\title{
Una experiencia de economía social y solidaria: La Asociación de Agentes Comunales de Salud Celendín. Cajamarca
}

Recibido: 03/10/2016

Aprobado: 14/11/2016

\author{
John García Saavedra, Cristhofer Zavala Bocinovich, \\ Rosman Bustamante Chávez, Héctor Calderón Tambra' \\ Universidad Nacional Mayor de San Marcos \\ < bchrosman@gmail.com >
}

\begin{abstract}
RESUMEN
Este proyecto de investigación-acción tiene como objetivo mostrar evidencia empírica de economía social, solidaria, popular y comunitaria en la Asociación de Agentes Comunales de Salud Celendín (Acomsac), de manera específica en el sector de producción apícola. Esta experiencia asociativa ubicada en el distrito de Celendín perteneciente a la provincia de Celendín en la región Cajamarca, es creada en 1982 con vigencia hasta la actualidad. Para la verificación de nuestro objetivo recurriremos a algunas propuestas teóricas dentro de la economía social, solidaria, popular, alternativa sustentada bajo valores de reciprocidad con énfasis en el valor de uso en vez del valor de cambio. Para ello, usaremos como herramientas de trabajo el análisis documental y bibliográfico, la observación y registro, entrevistas semidirigidas a actores de la asociación y de las instituciones (productores y usuarios), y preguntas abiertas.
\end{abstract}

Palabras clave: Agentes comunales de salud, sistema organizativo, autogestión.

\section{Association of Community Health Agents Celendin: an experience of social and solidarity economy. Cajamarca}

\begin{abstract}
This Research-Action Project aims to show empirical evidence of social, solidarity, popular and community economy in the Association of Community Health Agents Celendin (ACOMSAC), specifically in the sector of Beekeeping Production. This associative experience located in the district of Celendín belonging to the province of Celendin in the Cajamarca region was created in 1982 and is valid until today. For the verification of our objective we will resort to some theoretical proposals within the social economy, solidarity, popular, alternative sustained under values of reciprocity with emphasis on use value instead of exchange value. To do this, we will use as a working tool the documentary and bibliographic analysis, observation and registration, semi-directed interviews with actors of the association and institutions (producers and users), and open-ended questions.
\end{abstract}

KEYwORDs: Community health agents, organizational system, self-management.

$\overline{1 \quad \text { Estudiantes del } 8^{\circ}}$ ciclo de Sociología en la Universidad Nacional Mayor de San Marcos. Correo coordinador: < bchrosman@gmail.com > 


\section{Introducción}

$\square$ ste proyecto de investigación-acción tiene como objetivo mostrar evidencia empírica de economía social, solidaria, popular y comunitaria en la Asociación de Agentes Comunales de Salud Celendín (Acomsac), de manera específica en el sector de producción apícola. Esta experiencia asociativa ubicada en el distrito de Celendín, perteneciente a la provincia de Celendín, en la región Cajamarca, es creada en 1982 con vigencia hasta la actualidad. Para la verificación de nuestro objetivo recurriremos a algunas propuestas teóricas dentro de la economía social, solidaria, popular, alternativa sustentada bajo valores de reciprocidad con énfasis en el valor de uso en vez del valor de cambio. Para ello, usaremos como herramientas de trabajo el análisis documental y bibliográfico, la observación y registro, entrevistas semidirigidas a actores de la asociación y de las instituciones (productores y usuarios), y preguntas abiertas. Anotamos que la estructura de la investigación se desarrollará en cuatro secciones principales: recuperación de memoria o historia de la experiencia, relaciones con actores y agentes de su entorno, organización y conflictos internos, y las perspectivas o escenarios de futuro.

Es menester advertir de la muy escasa literatura respecto a la producción apícola de carácter asociativo lo cual deviene en una de las principales limitaciones sobre el tema de investigación.

Esta investigación se hizo gracias al apoyo y la hospitalidad de los agentes comunales a quienes afortunadamente pudimos encontrar en su II Asamblea Anual de la Asociación, a las Hermanas de la Congregación Las Carmelitas quienes nos brindaron el alojamiento por los días de nuestra estancia en Celendín, a la ONG Grufides por el apoyo económico que de modo desprendido nos otorgaron, a la PIC y a su presidente Milton Sánchez por facilitarnos los contactos e información previa y durante nuestro viaje. Dejamos constancia que no es el único apoyo que hemos recibido.

También damos nuestro reconocimiento a quienes no son mencionados en este corto agradecimiento, pero que su apoyo ha sido decisivo para nuestra tarea emprendida.

\section{Recuperación de la memoria o historia de la experiencia}

El que no vive para servir, no sirve para vivir. Vida saludable para todos. Acomsac (2009).

La frase del título pertenece a Robert Baden-Powell, atribuida a la Madre Teresa de Calcuta. Lema que resume y representa perfectamente la función y el objetivo que realiza Acomsac, Cajamarca.

Acomsac, con ubicación en la región de Cajamarca, provincia de Celendín, los Comités de Salud Locales se encuentran distribuidos y ubicados entre las zonas de 2,000 msnm a 4,000 msnm en los caseríos de Chumuch, Miguel Iglesias, Sorochuco, Huasmin, José Gálvez, Celendín, Utco, Cortegana y Pallan.Tiene su génesis en el año de 1982, como antecedente la iniciativa e impulso principal de su creación a la hermana María del Carmen Hernández Hernández de la congregación religiosa Hermanas Carmelitas de Vedruna, en menor medida pero de suma importancia al doctor Eduardo Díaz Abanto y al cura Ántero Mantaca Peralta. Estos tres personajes son considerados los fundadores de la organización.

La Acomsac es una organización civil cristiana católica sin fines de lucro que promociona y difunde la organización democrática y solidaria de la población rural para la defensa de la salud y la vida. Está conformada por campesinos, quienes son elegidos por su comunidad para asumir la representatividad y liderazgo, a la par asumir la responsabilidad y cumplir con el rol de nexo directo entre los agentes comunales de salud y el comité de salud local de dicha comunidad, tienen como objetivo velar por la salud de los integrantes de su comunidad.

Desde su fundación (1982) hasta la actualidad, el proceso histórico se divide en dos momentos:1) un primer momento que abarca desde el año de su génesis, 1982, hasta el año 2008, demarcado por la estabilidad económica y estabilidad institucional; 2) un segundo momento que se inicia en el ańo 2009 y se prolonga hasta la actualidad, en esta etapa se observa una crisis institucional en consecuencia directa de la dependencia económica de Acomsac-Europa. Y ante la crisis económica europea a manera de respuesta la búsqueda de nuevas fuentes de ingreso económico para que la institución pase de ser dependiente a ser autónoma y autogestionable. 


\section{Agentes comunales de salud}

A lo largo del análisis de nuestra historia se observa y se asume como uno de los ejes importantes de investigación a la religión y se concluye de esta la fuerte presencia de la iglesia católica en el desenvolvimiento social. No exento de ello, como ya mencionamos, la presencia de congregaciones misioneras, en especial la Congregación Religiosa de las Hermanas Carmelitas en Celendín, sumado a otros factores sociales, tendrá como iniciativa y consecuencia la creación de la Acomsac.

Las Hermanas Carmelitas de la Caridad de Vedruna son una congregación religiosa católica femenina de derecho pontificio, fundada por la religiosa espańola Joaquina de Vedruna en Vich (oficialmente y en catalán Vic, es un municipio español, capital de la comarca de Osona, situado en la provincia de Barcelona, comunidad autónoma de Cataluña), el 26 de febrero de 1826. Las Carmelitas se dedican especialmente a la educación cristiana de la juventud, en las escuelas y catequesis. Además desempeñan labores de asistencia hospitalaria. Sumado a lo anterior acompañan los procesos de crecimiento y de implicación social alentando talleres, cooperativas, grupos cristianos, preparación de catequistas y siempre en la reflexión y diálogo con la realidad desde planteamientos más o menos cercanos a la teología de la liberación. Joaquina de Vedruna, el 6 de enero de 1826, funda la Congregación Cristiana Católica de las Hermanas Carmelitas teniendo como fin la dedicación a la educación de la juventud y a la asistencia de los enfermos. Para su proyecto recibió la ayuda del religioso capuchino (Orden de los Hermanos Menores Capuchinos) Stefano da Olot Maturó y la aprobación del obispo de Vich, Pablo Corcuera. El día de la fundación fue el 26 de febrero de 1826 en la casa de la misma Vedruna en Vich, llamada el Manso Escorial.

Acomsac. Celendín en la década de los 80 disponía de tres centros de atención de salud para el total de la población: el Centro de Salud Celendín, el Puesto de Salud de Sucre y Miguel Iglesias. Sumado a esta adversidad el cuerpo médico que se disponía para la atención en salud era de un médico y cuatro enfermeros. La situación en atención médica era eminentemente caótica.

Visto esto y ante un concurrir frecuente de las zonas rurales y urbano periféricas por parte de las Hermanas Carmelitas de la Caridad de Vedruna, quienes comenzaron a visitar las comunidades con la misión y preocupación de la formación humana y cristiana, observaron esta carencia e idearon una estrategia ante este gran problema que aquejaba a los Shilicos (originarios de Celendín). Esta estrategia consistía en la capacitación en salud de primeros auxilios a los pobladores de las comunidades para que así en las comunidades se tenga a disposición una atención primaria de salud.

Es así que por iniciativa de la hermana María del Carmen Hernández Hernández, el doctor Eduardo Díaz Abanto y el cura Ántero Mantaca Peralta se funda en el año de 1982 la Acomsac. A partir de esa fecha se comenzó a promocionar que las comunidades envíen un representante para que este fuese capacitado.

La capacitación que reciben los representantes de cada comunidad es de dos ańos y tiene como primer objetivo promocionar la salud integral, fortalecer la vida y salud de la población rural y urbana periférica. Acomsac cuenta con nueve promociones de agentes comunales de salud.

\begin{tabular}{lc}
\hline PROMOCIÓN & NÚMERO DE PARTICIPANTES \\
\hline $1983-1984$ & 22 \\
\hline $1986-1987$ & 30 \\
\hline $1989-1990$ & 17 \\
\hline $1992-1993$ & 32 \\
\hline $1994-1995$ & 41 \\
\hline $1997-1998$ & 38 \\
\hline $2000-2001$ & 39 \\
\hline $2003-2004$ & 45 \\
\hline $2008-2009$ & 15 \\
\hline
\end{tabular}

Fuente ACOMSAC

Según la Acomsac, los agentes comunales de salud tienen como principales funciones:

La promoción y difusión de la organización democrática y solidaria de la población rural para la defensa de la salud y la vida, tratar de elevar la calidad de vida de la población que está ubicada en el ámbito de nuestro trabajo, especialmente salud, alimentación y medio ambiente: Promover la superación cultural, espiritual ;atención de primeros auxilios, atender a los pacientes que presentan casos sencillos; educar a la población en materia de salud para prevenir enfermedades; realización de actividades de apoyo para el desarrollo comunal, saneamiento básico, huertos familiares, crianza de animales, reforestación, cuidado del medio ambiente y mejora de los ambientes de preparación de alimentos; mantener en constante vigilancia a la comunidad, realizar constantes seguimientos de caso en las comunida- 
des y; luchar para hacer la vida más humana, trabajo comunal de salud "hacer que la vida humana sea cada vez más humana”.

Su accionar laboral ha contado con el apoyo de diversas organizaciones: Municipalidad de Celendín, Gobierno Regional de Cajamarca, Foncodes, Aprisabac, Generalitat Valenciana, a esto el rol que asumen las Hermanas era el de la elaboración de proyectos y el asesoramiento de la organización bajo la dirección de la Hermana María del Carmen Hernández Hernández quien tiene nacionalidad española sumado a esto se tiene como sede de la Hermandad de las Hermanas Carmelitas de la Caridad de Vedruna a España. El financiamiento que se recibía como apoyo para las actividades de la organización en su gran mayoría venía de instituciones españolas, por la relación directa que tiene esta hermandad.

El trabajo de los Agentes Comunales de Salud es el siguiente: 1982-1991, el trabajo fue enfocado en capacitar a los Agentes de Salud para poder generar las redes de trabajo, Local Comunal de Salud - Agentes de Salud Celendín; 1992, el cólera llega a Cajamarca, comienzan las labores intensas en salubridad. Agentes Comunales comienzan a desempeñar labores más intensas, tanto de prevención (charlas informativas) y labores en saneamiento de los hogares; 1993-1994, atención medica directa a niños desnutridos de tercer grado de primaria con el apoyo financiero de Foncodes. Asimismo, se trabajó como Aprisabac en la construcción de sistemas de agua potable para 16 comunidades, 9 de Huasmin y 7 de Celendín, igualmente se ayudó en la construcción de agua potable de Unión del Norte, Alto Perú y el Sauce;1995-2001 Continuidad en el trabajo con niños desnutridos con el apoyo de Generalitat Valenciana, promoviendo la preparación y el consumo de harinas balanceadas a base de cereales y menestras, además se fomentó la crianza de animales menores: cuyes, conejos, ovinos, trabajo en apicultura, piscigranja, huertos familiares y comunales así como la siembra de árboles frutales de acuerdo a la zona;20022008 se trabaja para mejorar los ambientes donde se preparan los alimentos, ya que de esto depende mucho la salud de la familia y así poder disminuir la incidencia de enfermedades. Se priorizo también la reforestación con plantas forestales en 58 comunidades del amito provincial;2008-2009, se continúa trabajando en actividades para el mejoramiento de la dieta familiar en las comunidades de los Agentes Comunales de Salud (Acomsac, 2009).

\section{Acomsac, en búsqueda de la autonomía y autogestión}

Desde sus cimientos, como antes ya fue visto (19822008), Acomsac se encuentra estrechamente ligado a la Iglesia católica, específicamente con las Hermanas Carmelitas de la caridad de Vedruna. A lo largo del tiempo la relación de trabajo Iglesia - Agente Comunal se ha desarrollado de forma conjunta. Por un lado las Hermanas Carmelitas junto a un grupo de trabajadores realizan la tarea de asesoramiento de la organización, la elaboración de proyectos y capacitaciones de los agentes para dar continuidad a su labor dentro de las comunidades, así mismo la búsqueda de financiamiento para estas y diversas actividades y por el otro lado (Agentes Comunales) encargados de realizar el trabajo dentro de las comunidades (prevención y saneamiento en salud) y a la par ser el nexo con la central de agentes. Esta división del trabajo a lo largo generara cierta dependencia, el Agente Comunal dentro de la organización adquiere un rol pasivo.El carácter que asumió erróneamente Acomsac en un primer momento fue el de no proyectarse en alguna actividad económica que le permitiese una estabilidad económica, no se consideró un proyecto a futuro que solventase o diera soporte a la organización mediante su propio trabajo.

Un segundo momento comienza en el año 2009, y tiene como punto de partida la crisis europea iniciada en el año 2008. La Hermana María del Carmen contaba con apoyo económico de instituciones que se encontraban en su gran mayoría en España, iniciada la crisis que azotó al viejo continente se hacía imposible el financiamiento por parte de estas instituciones por lo cual proyectos o futuros proyectos que se querían realizar quedaron truncados. Esta relación de dependencia económica comprometerá la estabilidad económica, organizacional y en proyección que tenía Acomsac. No obstante ante este problema los Agentes Comunales optaron por seguir en el camino de la lucha por la Salud pero sumaron a su causa un proyecto de sostenimiento económico sobre la base de sus pilares de reivindicación por la lucha de la vida. Se comenzará a desarrollar e idear un nuevo tipo de actividades con las cuales el objetivo que persiguen alcanzar es solventarse económicamente de manera autónoma y autogestionaria.

Las actividades económicas que desempeñan actualmente son la acupuntura, la apicultura, medicina natural y la artesanía. 
Organización y conflictos internos

La Acomsac, como se ha venido viendo, tuvo un papel protagónico desde su funcionamiento como promotores de salud en 1982. La principal virtud que se puede notar a primera vista es la preocupación que existe por las comunidades. El fomento de la organización de las comunidades para poder elegir un representante que pueda tener conocimientos básicos en temas de salud, ante la inoperancia o precariedad de este sistema, trajo consigo beneficios incalculables. La organización que propone la asociación puede no ser nueva, pero es efectiva en su deber principal de brindar salud. Además, la adaptación que existe de prácticas comunitarias es de gran valor para las metas de la misma asociación, al dar impulso a la organización de las comunidades y por otro lado, al brindar oportunidad a cualquier individuo de poder ser partícipe democráticamente de la asociación. Sin embargo, también hay que tener en cuenta que con el tiempo, los intereses, capacidades y posibilidades cambiaron, pero la organización no tuvo grandes cambios. Para poder entender el funcionamiento y el alcance que llega a tener la asociación es necesario identificar cuál es su sistema organizativo y cuáles son sus instancias de decisión.

\section{Sistema organizativo}

Para poder tener claro los fines y objetivos de la asociación no se puede dejar de lado el tema organizativo. De aquí parte una de las funciones principales: el dar una formación inherentemente ligada a valores de comunidad, de solidaridad y también ligados a la religión. Los alcances que esto llega a tener en los asociados y en el camino que traza la asociación serán revisados más adelante.

El vínculo entre asociación y comunidad es la base de todo el funcionamiento de la asociación. Sin embargo, existen otras instancias que se encuentran "fuera de la organización", pero que juegan un papel decisivo en la trayectoria de Acomsac, como son los comités locales de salud, los asesores permanentes y el equipo técnico.La asociación se encuentra compuesta de asociados, que representan en exclusividad a una comunidad. Es decir, cada comunidad se encuentra presente mediante un agente comunal, que es elegido en una asamblea de su comunidad. Además, cada sector que existe dentro de la asociación, como la apicultura, se encuentra orga- nizado internamente. Es importante remarcar este aspecto, ya que una de las principales variantes que pueden explicar o sostener el hecho de que la asociación se mueve y mantenga dentro de pautas solidarias de organización es el hecho de darle vida a la participación de los propios comuneros en sus comunidades y en la toma de decisiones de la asociación.

$\mathrm{El}$ agente comunal tiene el deber no solo de aprovechar y ejecutar la misión que la comunidad le encarga al escogerlo, sino que también esta oportunidad le brinda capacidades que puede aprovechar para su propia vida. Así, no es de extrańar entre los propios apicultores que aparte de producir miel para la asociación, ya posean sus propios cajones para el trato de las abejas y la miel. Es de subrayar el hecho de que muchos de los casos analizados por medio de entrevistas dieran como resultado que muchos de los comuneros que son elegidos como agentes por su comunidad no tengan estudios completos, y que sostengan que la asociación les brindó una oportunidad que no la hubieran tenido de otra forma.

Además, que se tiene una clara alusión a que este tipo de actividad económica que desarrollan se encuentra entrelazada al trabajo, la solidaridad y la oportunidad que brinda a todo comunero; así, uno de los entrevistados menciona: "La economía solidaria es una forma de ayudar a trabajar a los que no tienen muchas oportunidades. Da oportunidades" (Entrevista a Santiago Chaupe). Sin embargo, tampoco se puede obviar que las principales razones de la adhesión a la asociación giran en torno a la posibilidad que esta brindaba para poder ayudar a la comunidad mediante el agente comunal y sus comités locales de salud. Diferentes entrevistados mencionan en este sentido, que las ganas de poder aportar a su comunidad en algo que necesitaban con urgencia, como es el sector salud, les brindo el aliento para asumir la labor de capacitarse. De acuerdo a esto, el recojo de información también nos pudo brindar pistas acerca de cómo es que el agente comunal goza de cierta movilidad social o simbólica con las nuevas capacidades adquiridas. Tampoco se puede dejar pasar por alto que muchos ex-asociados, en el sentido de aprovechar las oportunidades de capacitación que la asociación brindaba, lo utilizaron como un trampolín. Una de las principales carencias que tiene la asociación hoy es que no existen nuevas promociones desde el 2009. Esto ligado a la ya explicada crisis espańola y la insuficiencia en financiación de proyectos, trajo consigo una nueva mira de la asociación, que necesaria- 
mente se vincula a la autogestión y a la "sobrevivencia del grupo". Los ingresos prácticamente solo sirven para mantener el local e implementos muy básicos.

Es de apreciar que la no vinculación, expresada en las nulas promociones desde el 2009, con más comunidades.

\section{Instancias de decisión}

Es importante señalar, antes de ingresar en el tema, que los comités locales de salud y los asesores permanentes son de gran importancia en la propia asociación. Los primeros por jugar un rol de apoyo al agente comunal. Los comités locales de salud, además, también tienen una organización interna y se encuentran en constante coordinación con el agente comunal.

Por el lado de los asesores permanentes, cumplen una función de apoyo a la asociación, que como ya se explicó, es exclusiva para socios comuneros. Esos asesores permanentes cumplen un papel protagónico en el rumbo de la asociación. Son tres partes que lo conforman:

- La congregación de las hermanas Carmelitas, que como fundadoras siguen teniendo una gran injerencia en la asociación

- El equipo técnico, que se encarga sobre todo de algunas capacitaciones y de la formulación de proyectos. Es importante el señalar también, que este equipo recibe un salario por sus labores.

- La enfermera, que tiene un papel menos significativo que los demás, pero mantiene su apoyo sobre todo en el tema consultivo.

La instancia donde se deciden los acuerdos y se discute la dirección de la asociación son las asambleas anuales. Sin embargo, cada espacio dentro de la asociación tiene su propia organización, fundada por la autogestión que tiene cada uno de estos espacios. Así, el sector apícola tiene su propio presidente y tesorero. Lo mismo ocurre con los comités locales de salud, con los acupunturistas, las artesanas, etc. Una entrevistada del sector artesanal decía: "Cada grupo ve cómo se mantiene, casi todo lo que ganamos se queda en nuestro taller. De 30 soles que ganamos en una venta, 25 se quedan con nosotros para comprar nuestras cosas y 5 van para la asociación".

De esta misma manera, el sector apícola subsiste por sí, siendo la segunda actividad con mayores retribuciones para la asociación. De 50 kilos producidos de miel por un asociado, 25 van para la asociación y lo demás para él. Sin embargo, es casi un hecho obligatorio que esta parte que queda en poder del asociado sea vendida a la misma asociación. De esta manera se evita el trabajo de venderla y colabora con la asociación.

La asamblea general se da en el transcurso del 4 de Junio, fecha donde se celebra el día del agente comunal. En la asamblea del 2016, en la cual pudimos ser partícipes, se pudo evidenciar que existe un gran peso de parte de los asesores permanentes, sobre todo en el caso de las hermanas Carmelitas. La asociación tiene en claro su principios de fundación, basados en los valores cristianos. Y aquí se pudo presenciar que existe una clara jerarquía, sino material, simbólica, entre los asociados y las hermanas Carmelitas. Jerarquía que no niega los lazos de comunidad y solidaridad que existe dentro de la asociación, pero que presenta un hito peculiar.

Es de suma importancia el señalar que los propios asociados coinciden en que se necesita una nueva promoción. La renovación generacional es una necesidad que va ligada a una renovación organizativa y de aspiraciones. La llegada de hospitales o postas y colegios a las comunidades que antes no los tenían tuvieron repercusión en las posibilidades que tenía la asociación de crecimiento y afianzamiento. "Yo quiero que mis hijos sean profesionales. Uno ya está yendo al colegio y los otros dos están estudiando en Lima”, mencionaba Santiago Chaupe. Las perspectivas cambian al igual que las oportunidades y ser un agente comunal paso a un plano distinto al que pudo ocupar en épocas anteriores.

Esto no niega, sin embargo, que la asociación se siga rigiendo por la solidaridad y los valores que brinda el sistema comunitario. Tampoco se puede obviar el papel que juega la religión como base de la organización. Sin embargo, dada la situación de la asociación, y dentro de sus posibilidades se tiene que tener en cuenta que hay dos niveles que se podrían tratar. El primero en el cual podemos vislumbrar el sistema organizativo, que aboga por el trato igualitario, por la posibilidad de que cada miembro pueda ser partícipe de los ámbitos de decisión y de las tareas que se dictaminan. Sin embargo, el hecho de que la miel se encuentre a un precio más elevado que en mercado local, juega en contra de estas pautas mencionadas. La miel no vuelve a las comunidades, se queda en Celendín y generalmente es comprada por visitantes. En este nivel, existe una deficiencia, justificada por las pocas herramientas productivas con las que cuenta la asociación, y que obligan a que los precios sean elevados. 


\section{Relación con el entorno}

Para este aspecto de la investigación acción, la información recabada de las instituciones gubernamentales y no gubernamentales, planteamos relaciones de Acomsac con: Caseríos, Plataforma Interinstitucional Celendina, Gobierno Regional, Entidad Religiosa (Congregación Hermanas Carmelitas), Universidades, Municipalidad Provincial, Empresa local y usuario/ consumidor.

La información recogida nos permite identificar al Conflicto Socioambiental del Proyecto Minero Conga, conocido como conflicto "Conga", como el principal indicador y eje de coordinación y ejecución de medidas conjuntas, tanto en lo social, en lo político, en lo económico y hasta en lo cultural. Esto es, el surgimiento del conflicto conga se convierte en el elemento que determina la consolidación de las relaciones con el entorno.

\section{Caseríos}

\subsection{Promotores de salud como primer rol}

El rol de promotores de salud les permite mantener contacto directo con las comunidades y sus problemáticas. La información de la asociación a la comunidad y viceversa se canaliza a través de los promotores de salud, quienes gozan de credibilidad y confianza por el papel que cumple cubriendo el vacío del estado y porque es un trabajo de servicio no remunerado y voluntario.

\subsection{Agentes politicos/organizativos como segundo rol}

El papel primigenio de promotores de salud que cumplían los agentes comunales es rebasado a partir del conflicto Conga. La legitimidad que poseen al ser elegidos en asamblea comunal auspicia el papel político que desempeñan desde comienzos del conflicto Conga, es benéfica para convocar y hacer llegar la información, las decisiones, la postura, y las acciones a ejecutar de la Asociación respecto al conflicto. Esto les permitió durante el estallido del conflicto mantener al tanto y ser quienes convocaran a asambleas informativas cuando otras instituciones defensoras de los recursos hídricos, le agua y del medio ambiente, lo solicitaran.
La importancia del papel político y organizativo de los agentes comunales comienza cuando como Acomsac son los primeros en sacar un pronunciamiento denunciando los riesgos que puede traer la ejecución del proyecto Conga, antes que ninguna institución visibilizara el problema.

\subsection{Agentes económicos como tercer rol}

La Asociación en la actualidad es productora, dentro del sector apícola de la asociación, de miel de abeja bajo la dinámica descentralizada la cual consta de que cada agente comunal, interesado en la producción de miel, cultive mínimo un cajón de abejas en su comunidad y los litros de miel producida los vende ya sea a la misma asociación (que por ser socios prefieren hacerlo) $\mathrm{u}$ a otros compradores. De ese dinero generado el cincuenta por ciento es para el productor y cincuenta por ciento es para a Asociación.

Esta actividad económica, nueva iniciativa dentro de la asociación, con el dinero generado beneficia directamente a las familias de los agentes comunales y también al vecindario, aunque en mínima proporción, cuando la miel es utilizada para curar enfermedades comunes del lugar como toz generalmente.

\section{Plataforma interinstitucional celendina}

La PIC se crea en el 2012 por el Conflicto Conga, y Acomsac es miembro fundador de la misma Plataforma, por lo que las relaciones durante el conflicto fueron claves para el desenvolvimiento de los planes y la ejecución de las estrategias de lucha. El local de Acomsac, además de ser el lugar donde se alojaban las delegaciones de las comunidades que llegaban a la provincia de Celendín a protestar, también sirvió de lugar de coordinación y planeamiento de las acciones que se ejecutaron durante el conflicto, entonces la PIC liderado por su presidente el Sr. Milton Sánchez entra en una indispensable coordinación con los agentes y la asociación en general para conjuntamente sostener la resistencia emprendida.

\section{Gobierno Regional}

Durante el conflicto las coordinaciones entre el gobierno regional y Acomsac se han dado en tanto medidas 
de lucha y apoya a ellas, sin embargo en la actualidad no existe mayor relación, es más dentro de las políticas dentro del Nuevo Modelo de Desarrollo, el gobierno regional de Cajamarca no contempla trabajar con asociaciones que no estén inscritas en registros públicos (formalizadas) ni con asociaciones que no poseen visión empresarial, y los dos requisitos no los tiene Acmosac.

\section{Usuario/consumidor}

El usuario o consumidor, de la miel de abeja específicamente, mantiene una relación bajo términos de calidad y garantía. Si bien el mismo producto que ofrece la Asociación cuesta más (15 soles) que el producto que se ofrece en el mercado de abastos de Celendín (10 soles), los usuarios prefieren el producto de la Asociación porque consideran que es miel pura.

\section{Gobierno provincial}

Las relaciones en la actualidad son circunstanciales en relación a cuestiones operativas más no institucionales, dígase, la municipalidad brinda apoyo con andamios y toldos para la realización de ferias productivas que organiza Acmosac en una de las cuales tuvimos la oportunidad nos encontramos presentes. El poyo depende de la filiación política que tenga el régimen de gobierno provincial, a decir, durante el conflicto Conga el alcalde de la provincia estuvo del lado opuesto a las demandas que Acmosac y la PIC emprendieron en conjunto con las comunidades campesinas afectadas por el proyecto. Es más, a municipalidad en ese entonces enviaba espías al local de Acmosac (donde se planificaba los planes de lucha).nos indica la Hna. Leonor (55) quien prefiere llamarse Asesora en vez de asumir un nombre jerárquico dentro de la Asociación.

\section{Entidad religiosa: Congregación Hermanas Carmelitas de la Caridad de Verdruna}

Por lo que se ha menciona párrafos antes, las Hnas. Carmelitas son las fundadoras de la Asociación, por lo que una primer relación que existe entre esta y la Congregación está dada por lazos de dependencia: La asociación sigue el ritmo que le dan las Hermanas, en ese sentido las coordinaciones institucionales lo hacen principalmente las Hermanas, quienes también han sido quienes a lo largo del tiempo han conseguido financiamiento para la organización; a decir de los socios, se puede notar un cierto grado de pasividad respecto a la dinámica que sigue la Asociación, se ciñen a lo que la directiva(principalmente las madres consigan), no se ve una iniciativa mayor a la de ejecutar lo que se dispone por parte de la directiva.

Además se puede identificar una relación basada en valores: Solidaridad, no lucro, bien colectivo, preferencia por los pobres. Dado que la orientación religiosa de la congregación en mención es de tendencia clara de la Teología de la Liberación ${ }^{1}$

\section{Universidades}

$\mathrm{Si}$ bien se tiene convenio con un instituto local (IFFDP), en la actualidad tanto en el ámbito, local, regional y nacional no cuentan con ningún convenio institucional ni lazos institucionales con ninguna universidad. Sin embargo a decir de la dirigencia de Acmosac se espera de las universidades generen sensibilidad en cuanto a la problemática ambiental que reivindica la Asociación como tal en su papel organizativo que juega dentro de la sociedad celendina, y entablar lazos institucionales para desarrollar actividades conjuntas como talleres, cursos, charlas y/o proyectos que fortalezcan el desenvolvimiento de la Asociación y Universidad.

\section{Perspectivas o escenarios de futuro}

Analizar los escenarios a futuro que los mismos actores de una organización se proyectan puede servir a que sea más visible el diferenciar entre una perspectiva que ve desde el "mercado capitalista" de aquella que ve desde la "economía social". De manera muy resumida la primera perspectiva parte de la premisa de que al no hallar otra alternativa posible a la lógica de mercado capitalista, todo tipo de emprendimiento económico-social debe de "adaptarse" al mercado, es decir no se acepta la posibi-

1 La teología de la liberación es una corriente teológica cristiana integrada por varias vertientes católicas y protestantes, nacida en América Latina tras la aparición de las Comunidades Eclesiales de Base, el Concilio Vaticano II y la Conferencia de Medellín (Colombia, 1968), que se caracteriza por considerar que el Evangelio exige la opción preferencial por los pobres y por recurrir a las ciencias humanas y sociales para definir las formas en que debe realizarse aquella opción. Tiene como pioneros a al Sacerdote peruano Gustavo Gutiérrez y al Brasileño ex pastor presbiteriano y educador Rubem Alves. 
lidad de que se piense en formar organizaciones, emprendimientos, cooperativas, o hasta mismas empresas que se manejan con otra racionalidad distinta a la capitalista y que entonces más bien representen alternativas concretas al modelo de mercado hegemónico; de lado contrario desde la otra perspectiva surgen teorías que intentan demostrar que es posible una apuesta teórica y política que haga frente a la actual racionalidad capitalista hegemónica (Muñoz, 2011). Es por ello que se hace necesario partir de los hechos a la teoría y no al revés, y precisamente al ir conociendo las propias perspectivas de los actores involucrados en la Asociación de Agentes Comunales de Salud Celendín (Acmosac) se podrá vislumbrar qué perspectiva podrá ser considerada la más adecuada a esta realidad empírica y concreta.

Desde esta pequeña indagación también se podrá aportar al debate de "cómo clasificar" este tipo de experiencias. Quijano sintetiza las distintas aproximaciones a estas experiencias en dos grandes vertientes, por un lado la economía solidaria (y su institución central, la cooperativa) y la economía popular:

... en el caso de las cooperativas están más presentes las reglas del mercado y del salario, y la reciprocidad opera como desde fuera de las relaciones de trabajo, por una decisión consciente de los miembros o, por lo menos, de la minoría dirigente y aceptada por la mayoría. Por eso, seguramente, cuando esa conciencia no existe, o cuando se estrella contra situaciones desventajosas en la materialidad de las relaciones de trabajo y de distribución del producto, las cooperativas se desintegran o se transforman en empresas convencionales para reproducirse y crecer. En cambio en el heterogéneo universo de las llamadas "organizaciones económicas populares", en muchas ciudades latinoamericanas, es la materialidad misma de las relaciones sociales la que requiere, obliga si se prefiere, a la solidaridad. En otros términos, es el hecho de que la reciprocidad sea la naturaleza misma de las relaciones sociales lo que entrańa la práctica de la solidaridad, incluso quizá al margen, si no necesariamente en contra, de la conciencia política y de la ética social formal de los miembros. (Quijano, 2011).

Como vemos la diferencia central reside en la condición de "conciencia" de las relaciones de solidaridad, las experiencias de economía popular "se ubican ideológica y políticamente, de modo explícito, en contraposición al capitalismo" (Quijano, 2011). En cambio la solidaridad en las experiencias de economía popular radica en la materialidad de sus relaciones.
Volviendo al caso específico que tratamos, la experiencia de Acmosac, cabe resaltar que para aproximarnos al tema de sus perspectivas o escenarios de futuro que se proponen los mismos actores se hizo necesario dividir nuestra principal herramienta que utilizamos - la entrevista a profundidad - en tres segmentos: un primer segmento en el que se tratan las perspectivas a futuro desde un corte temporal (objetivos inmediatos, a corto y largo plazo), un segundo segmento en el que se trata el tema desde un corte espacial-geográfico (visión a futuro en la localidad, región, país, redes) y un tercer segmento en el que se trataron los valores e ideales que le dan sustento a estos posibles escenarios y la visión de futuro que construyen los actores.

\section{Autogestión colectiva como reto}

Acomsac surge como una respuesta a la inoperancia y la poca cobertura del Estado en la prestación de servicios de salud. En un primer momento esta asociación se financiaba por apoyo de la orden religiosa "Las Carmelitas", esta orden conseguía la mayoría de sus fondos de distintas instituciones en España. Después de la crisis económica en el año 2009 en España se fue reduciendo el financiamiento para las actividades y el funcionamiento de Acomsac. Esta situación forzó a la asociación a buscar otras formas más estables de financiamiento, en este momento empezó la carrera por hacer "rentable" la asociación, los miembros decidieron enrumbarse en el reto de la "autogestión", este escenario se resume muy bien en una frase de uno de los socios: "nos habian dado pescadito, pero no nos habian enseñado a pescar, recién estamos aprendiendo ello". Ahora entonces sumado al tema de la promoción de la salud, los socios han incursionado en distintas actividades productivas.

Hasta el momento los socios han aprendido y vienen trabajando en 3 actividades principales: productos apícolas, medicina natural y la acupuntura, Se suma también la venta de prendas de vestir, recuerdos y artesanías. Sin embargo, a pesar de estos notables esfuerzos por volver rentable la asociación esto no se ha logrado y sigue significando un reto para los socios. Entonces surge la pregunta ¿Por qué permanecen los socios en Acomsac? Como veíamos más atrás, para Quijano, las relaciones solidarias permanecen porque estas se desenvuelven de manera casi inconsciente en la asociación, se corroboraría esta visión en que precisamente por esta 
condición inconsciente de las relaciones solidarias es que la asociación no deviene en una empresa después de las "situaciones desventajosas" (Quijano, 2011) por las que pasa - sin embargo como veremos más adelante es clara la posición política consciente de los miembros de Acomsac-, esta explicación de Quijano nos sirve hasta cierto punto. Sumamos a esto dos factores. Primero, el vínculo de pertenencia y el sentido de afiliación que ha sido interiorizado por la mayoría de los socios. Segundo, la búsqueda por "movilidad social", en el sentido del "status" que obtienen al adquirir estos conocimientos que escapan del conocimiento cotidiano en las comunidades en donde viven los socios, ellos son conscientes de como este aprendizaje les posibilita nuevas oportunidades tanto en su proyecto de vida como en sus comunidades (aunque en algunos casos también se generan conflictos).Con esto no queremos decir que sea el interés individual lo que sostiene la asociación, es más este es un tanto relevante comparado con las relaciones solidarias y el sentido de afiliación de los socios con ACOMSAC, aun así es necesario identificar todos los factores y relacionar como estos se superponen entre sí.

Los socios han asumido que esta situación es insuficiente para sostener la asociación, sumado a las actividades en las que vienen trabajando, buscan expandir esta gama de actividades así como también buscan expandir sus mercados. Los mismos socios vienen trabajando en dos posibles salidas a este problema de rentabilidad, para estas tareas vienen formando redes y reciben ayuda de otras instituciones, por ejemplo la consultora GEASSED, que les brinda apoyo principalmente a través de charlas y capacitación técnica. En el caso de la búsqueda de nuevas actividades, ven como central el tema de la "diversificación productiva", sobre todo productos agrícolas, vienen recibiendo charlas y haciendo algunos pequeños trabajos con productos como flores, paltas, papa, quinua, entre otros. Por el lado de la exportación, esta idea no está del todo clara, falta aún muchos temas e inconvenientes con respecto a la exportación por precisar; sin embargo si tienen el total interés por salir sobre todo a mercados internacionales.

Un punto clave para que marche la autogestión reside en el tema de cómo está organizada la asociación, con respecto a esto Acomsac no ha tenido cambios sustanciales en la estructura organizativa, se han venido manejando con la lógica de que por cada comunidad solo puede haber un socio. Este mecanismo puede ser un tanto contraproducente por el hecho de que hace ya
6 años que no sacan una nueva promoción de socios y además que la tendencia de los mismos es a ir disminuyendo en número, es más, los propios socios consideran que uno de los temas más urgentes a resolver es el de poder sacar una nueva promoción de socios. Por esto se debe asumir como un nuevo reto el tema de la "reorganización" de sus normas y estructuras de base, una humilde sugerencia sería la de "abrir la organización", en el sentido en que se aumente a más de uno el número de socios que pueden ser de la misma comunidad, esto evaluando siempre dentro de sus propias posibilidades, principios y proyecciones.

\section{La lucha por el medio ambiente}

Como ya se ha hecho mención, Acomsac realiza sus primero trabajos exclusivamente abocados a la promoción y tratamiento de la salud, esto implicaba un rol central de la asociación en las comunidades a las que llegaba, en algunos casos se superaba la labor en salud y se apoyaban en otras actividades. Conforme el estado llegaba a estas comunidades Acomsac perdía centralidad en estas, en cierto sentido fueron desplazados en algunas labores, también en el tema de salud. No sería preciso afirmar que hubo una competencia entre el estado y esta asociación, más bien hubo distintas maneras de relacionarse, algunas veces en coordinación otras veces simplemente se tuvo que limitar la labor en temas menores de salud, de repente porque había llegado una posta o algún médico. Sin embargo siendo la promoción de la salud un tema central de la asociación, este fue perdiendo centralidad en los objetivos y el discurso de la asociación, o en todo caso se vio como necesario sumar otros elementos.

Sucede en Celendín el conflicto de Conga y este es otro punto de inflexión en la historia de Acomsac. La asociación cumplió un rol importante en oposición al proyecto Conga, impulsó y formó parte de la PIC (plataforma interinstitucional celendina), de esta experiencia amplió el tema de la promoción de salud, ahora la centralidad estaba en la "defensa de la vida", que incluía el tema de la salud sumando la lucha por la defensa del medioambiente. Actualmente es crucial y está interiorizado la lucha medioambiental en la mayoría de socios: "nosotros queremos un ambiente limpio, no somos terroristas antimineros" nos decía uno de los socios, varios de ellos y también las monjas carmelitas nos confirmaban esta idea con sus propias palabras, es decir, su apuesta 
por defender el medioambiente es un "acto político consciente". Con respecto a este punto, cabe resaltar que a diferencia del tema de la salud, la consigna de "por defensa de la vida" — como nos decía una de las monjas carmelitas - puede resultar un tanto "ambiguo", esto sumado a la fuerte presencia de la iglesia católica (las monjas carmelitas y la parroquia de Celendín que trabaja constantemente con Acomsac) deja a la reflexión sobre qué otros matices puede tomar esta consigna en otros contextos en los que algunas posiciones institucionales de la iglesia católica se vean afectadas (como el tema del aborto, derechos sexuales, etc.).

Esta centralidad en la lucha medioambiental implica de alguna manera que los agentes comunales de salud pasen a ser también "agentes políticos", y cumplan cierto rol en la organización y transmisión de estas y otras consignas en las distintas comunidades, aunque esta idea no es tan aceptada por los socios, de hecho los socios entrevistados muestran un total rechazo al término "política", llegando algunos a afirmar que: "nosotros no hacemos política". Sin embargo desde una perspectiva de construcción del poder en pro de cierta transformación o consigna, se les podría catalogar en todo caso como "agentes de cambio". Sin embargo esta amplitud de sus consignas y su condición de agentes no significa que las "circunstancias" hayan "modelado" a Acomsac teniendo que adecuarse totalmente a estas, los valores centrales que le dan sentido a la asociación siguen tan vigentes como desde sus inicios, estos son principalmente la solidaridad, el trabajo, la opción por servir, entre otros valores ${ }^{2}$.

\section{Posibles escenarios a futuro}

Podemos dar cuenta del total cambio por el que ha pasado Acomsac, podemos dar cuenta de que en menor o mayor medida los mismos socios son conscientes de estos cambios sustanciales. Podemos concluir que actualmente Acomsac sigue afrontando dos escenarios distintos a lo que se plantearon en un inicio como organización y estos son asumidos como retos.

Por un lado el tema de la autogestión y la rentabilidad de la organización. En este punto es central el tema de la reorganización de Acomsac para abrir la asociación y que puedan sacar lo más antes posible

2 Cabe precisar que uno de los documentos para sus talleres de los socios trata exclusivamente sobre una sistematización de los valores principales en los que debe basarse la asociación. una nueva promoción, a más socios puede que sea más rentable la asociación. Sumado a esto existen otras posibilidades, algunas como el tema de la exportación, de la innovación en nuevos productos y la búsqueda de financiamiento en otras instituciones (redes con las distintas instancias de gobierno) ya vienen siendo trabajadas por los mismos socios ${ }^{3}$. Otro tema importante es el de la "productividad", es necesario que replanteen sus estructuras de costos y precios y redefinan bien los mercados a los que estarán dirigidos ${ }^{4}$.

Por el lado de la lucha por el medio ambiente, es notable el papel de Acomsac en Celendín en el conflicto de Conga, hasta el momento estas experiencias han servido también de soporte en la misma asociación, estas experiencias también unen a los mismos socios. Sin embargo, como mencionábamos, el tema de la salud ha sido en su mayoría asumido - en menor o mayor medida - por el Estado, sin embargo esta tarea puede seguir siendo relevante, en tanto se asuma un rol de "colaboración" entre la asociación y distintas instituciones estatales, por ejemplo, a través de los colegios o en las mismas postas se puede revalorar los conocimientos y prácticas tradicionales que están inmersos en la producción de miel, medicinas naturales y otros productos que elabora Acomsac. Entonces sí es posible un retorno a trabajar como central el tema de la salud, esto no debe implicar que descuiden la lucha por el medioambiente que han asumido, este tema se ha vuelto central en la asociación y en su relación de esta con la localidad de Celendín y Cajamarca. También es necesario que se amplíen las redes en pro del cuidado del medio ambiente, una estrategia podría ser que busquen la manera de ingresar a la agenda y al debate nacional con fuerza, lamentablemente el tema del cuidado del medio ambiente es reducido a los "conflictos socio-ambientales" y estos son vistos desde la capital como simples "manifestaciones antimineras", ¿Cómo hacer frente a estos agresivos estereotipos? Es un gran reto hasta el momento.Estos posibles escenarios a futuro son solo humildes conjeturas académicas que no pretenden soslayar el papel de "actores activos" de los socios miembros de ACOMSAC, finalmente son ellos quienes asumirán que decisiones tomar, siguiendo sus objetivos sin perder de vista los valores que le dan sentido a la asociación.

3 Esto no implica que se pierdan de vista los valores que le dan sentido a la organización.

4 Hoy en día es principalmente a un sector de clase media del mismo Celendín o turistas, muy pocos productos regresan a las comunidades. 


\section{Reflexiones finales: Niveles de la economía social y solidaria}

Como menciona Coraggio el reconocimiento de las economías populares y solidarias demandan la construcción de una nueva propuesta económica que logre superar tanto la economía neoclásica como la economía política (Coraggio, 2011), para esto se hace también necesario tener en cuenta la precisión y rigurosidad de conceptos, teorías y métodos que dicha superación implica, entendiendo que este trabajo académico se reconstruye en las distintas investigaciones empíricas. Partiendo de esta idea es necesario ir precisando algunas cuestiones.

Para seńalar que tal experiencia pueda ser considerada como una de economía social, solidaria o popular, la mayoría de autores ponen el énfasis en las relaciones de solidaridad que se despliegan al interior de la organización, resaltando temas como la autogestión (Santos \& Rodríguez, 2011), la centralidad del trabajo dentro de la organización por encima del capital (Coraggio, 2011), el peso de las relaciones de reciprocidad (Quijano, 2011), las estrategias desplegadas para la reproducción de la vida ampliada (Cabrera \& Vio, 2014), entre otros temas. Sin embargo, en muchos casos la centralidad en las cuestiones internas de la organización implica cierto "vacío" en los temas de "cómo se inserta una organización potencialmente solidaria en el mercado", tocaremos un ejemplo.Boaventura de Sousa Santos trata el tema de las cooperativas centrándose en su organización y sobre todo en la "autogestión", pone como ejemplo distintos casos en distintos países de este tipo de experiencias exitosas, uno de ellos es la experiencia del conjunto de cooperativas en Kerala (India):

Como lo demuestra el detallado estudio de caso de una cooperativa de productores de cigarros de Kerala, hecho por Issac, Franke y Raghavan (1998), los factores esenciales para sobrevivir frente a la competencia de las empresas capitalistas son una combinación de descentralización y colaboración entre cooperativas asociadas en red y la fidelidad al principio de participación democrática dentro de la cooperativa ${ }^{5}$. (Santos \& Rodríguez, 2011).

Al mencionar que es necesario señalar "cómo se inserta una organización potencialmente solidaria en el mercado" nos estamos refiriendo a identificar también como importante: los productos que se están comer-

5 Ambos resaltados dentro de la cita son nuestros. cializando o produciendo y su "valor moral" (si es el caso de una cooperativa o asociación productiva), los precios con los que van al mercado, el público específico al que está destinado dichos productos, y otros temas que en otras investigaciones se definirán mejor. En el caso de la cooperativa de Kerala, cabe preguntarnos ¿Qué tan solidaria puede resultar una cooperativa que comercialice con cigarros?.Con esto proponemos dos niveles para poder definir si tal experiencia es de economía social, solidaria o popular. Un primer nivel es el de la organización interna, se identificarán las relaciones de solidaridad que se desplieguen al interior de la organización. Un segundo nivel sería el de la "inserción en el mercado" 6 , y proponemos identificar 3 aspectos a tomar en cuenta (que pueden ser ampliados obviamente): los productos que se están comercializando o produciendo y su "valor moral", los precios con los que van al mercado y el público específico al que está destinado dichos productos.

En el caso de Acomsac, bajo el primer nivel de análisis que proponemos podemos evidenciar que la autogestión es central en la organización, esta ha sido asumida como un reto e implica el despliegue de relaciones de solidaridad entre los distintos socios, esto sumado a la promoción y defensa de la salud y la vida. Con respecto al segundo nivel que proponemos es necesario recalcar que estos tienen que ser desde ya problematizados, en el caso de Acomsac desagregaremos los 3 aspectos que proponemos analizar. Con respecto al primer punto, los productos que se ofrecen son de total calidad y son productos destinados a la mejora de la salud de las personas. Segundo, la mayoría de sus productos están muy por encima del precio normal en el mercado de su localidad, sin embargo esto se debe a la "poca productividad" y sus "altos costos de producción". Tercero, el costo alto de sus precios implica que la mayoría de sus compradores sean de clases medias o turistas, aunque no es que Acomsac lo plantee de esta manera. El tema de los precios resulta controversial, puesto que como decía Coraggio (2011), es necesario superar la economía neoclásica (neoliberales) y la economía política (corrientes marxistas), esto sumado a la pretensión de construir un nuevo paradigma que según Escobar tendría sus raíces en el "postestructuralismo" (Escobar, 2005). Ambas teorías han logrado condensar una "posición" clara en el debate sobre la formación

6 Aunque habría muchas otras formas de formar redes, no necesariamente habría que introducirse al mercado. 
de los precios, mientras que los neoliberales plantean que el precio es el resultado de las relaciones subjetivas que se dan en el mercado, desde Marx se ha sido muy enfático en precisar que es en el mismo proceso de producción donde la mercancía forma su valor, y el precio es solo relativo a este valor y al margen de ganancia que se puede obtener. Entonces, sería erróneo pretender soslayar este debate o simplemente plantearles críticas a estas teorías-críticas que siempre serán válidas- si se pretende construir un nuevo paradigma ¿Qué se puede proponer desde este nuevo paradigma con respecto a este punto? Así como este tema, estamos seguros que aún hay muchos por discutir y precisar, dejamos abierto el debate. Entonces podemos hacer una comparación de estos dos niveles propuestos con la distinción clásica entre el "valor de uso" y el "valor de cambio". Este pequeño análisis de cómo la experiencia de Acomsac se inserta en el mercado puede ser también abordado desde el "valor de cambio", nuestro énfasis en este punto radica en que como seńalábamos no se ha tratado este tema de manera conjunta con el tema del valor de uso. Consideramos que el investigador no puede dejar pasar aquello que observa, si en una experiencia se desplieguen tanto lógicas de autogestión como de intercambio tendría que abordarlas ambas para poder dar un análisis más riguroso y si presunciones sesgadas. Esto sin embargo, no significa que neguemos o soslayemos la importancia que tiene en Acomsac la autogestión y el valor de uso por encima del valor de cambio, como ya mencionábamos esta es una experiencia donde la salud es central, e incluso esta logra proyectarse a la idea de defensa de la vida. Ya dicho esto es preciso señalar que en distintos trabajos previos se soslaya más bien el tema de la inserción al mercado o el tema del valor de cambio. Consideramos pertinente el abordaje de ambos niveles y el consiguiente balance entre ambos, ¿Se puede dar una respuesta apriorística sobre qué pesa más, el valor de uso o el valor de cambio?7, partiendo de que en Acomsac sí existe un peso claro de la autogestión y la defensa y promoción de la salud y la vida, y por lo tanto se supera el ámbito del mercado, pues va más allá de la oferta y la demanda, o el intercambio de-

7 También cabría preguntarnos si se puede responder apriorísticamente si las experiencias de economías otras pueden ser alternativas al mercado capitalista o más bien pretenden integrarse a este, tema que debe ser precisado en cada experiencia concreta y bajo la pretensión de no caer en juicios de valor. En este camino de precisión teórica es válido la construcción de tipologías, un notable aporte sería la distinción entre economías solidarias y economías populares que presenta Quijano (2011). terminado por la expectativa subjetiva, ¿Pasará lo mismo con otras experiencias?, ¿Se hace necesario analizar los dos niveles propuestos y evaluar rigurosamente sin prejuicios sesgados?, dejamos abierto otro debate.

\section{Referencias bibliográficas}

Acomsac (2009). Forjando salud y desarrollo en Celendin.

Cabrera, M., \& Vio, M. (2014). Los hilos de la economía popular en la posconvertilidad. En M. C. Cabrera, \& M. Vio, La trama social de la economía popular. Espacio Editorial.

Coraggio, José Luis (2016). La economía social y solidaria (ESS): niveles y alcances de acción de sus actores. El papel de las universidades, en: Carlos Puig (Coordinador) Economía Social y Solidaria: conceptos, prácticas y politicas públicas, Bilbao: Hegoa Instituto de Estudios sobre Desarrollo y Cooperación Internacional de la Universidad del País Vasco.

Coraggio, J. L. (2011). Economía social y solidaria. Ediciones Ayba-Yala.

Escobar, A. (2005). El "postdesarrollo" como concepto y práctica social. En D. Mato, Políticas de economía, ambiente y sociedad en tiempos de globalización. Facultad de Ciencias Económicas y Sociales, Universidad Central de Venezuela.

Germaná, César (2016). La economía de la reciprocidad y el Buen vivir, en: Hanne Cottyn, Javier Jahncke, Luis Montoya, Ela Pérez, Mattes Tempelmann (Editores), Las luchas sociales por la tierra en América Latina. Un análisis histórico, comparativo y global, Lima: Seminario de Economía Social, Solidaria y Popular, Facultad de Ciencias Sociales, Universidad Nacional Mayor de San Marcos.

Quijano, A. (2011). ¿Sistemas alternativos de producción? En B. d. Santos, Producir para vivir. Fondo de Cultura Económica.

Marañón Pimentel, Boris (2016). Notas sobre la solidaridad económica y la descolonialidad del poder, en: Boris Marañón Pimentel (Coordinador), Políticas para la solidaridad económica y el Buen Vivir en México, Ciudad de México: Instituto de Investigaciones Económicas de la Universidad Nacional Autónoma de México.

MuÑoz, R. (2011). Alcances y desafíos de la economía popular urbana. En P. Urquieta, Ciudades en transformación. Plural editores.

Santos, B. d., \& Rodríguez, C. (2011). Para ampliar el canon de la producción. En B. d. Santos, Producir para vivir. Fondo de Cultura Económica. 